\title{
SISTEMAS DE SALUD Y SUBSIDIOS A LOS POBRES Un modelo de financiamiento de salud para el Perú
}

\author{
José Carlos Vera \\ INVESTIGADOR DE ESAN \\ MÁSTER EN DESARROLlo ECONÓMICO \\ jvera@esan.edu.pe
}

\begin{abstract}
Resumen
Desarrolla un modelo de financiamiento de servicios de salud pública que establece cuál sería el gasto en salud por persona que tendría que hacer el Estado según el paquete de servicios que desee ofrecer y la población que decida subsidiar. Con este objetivo propone un sistema de seguro de salud para toda la población, en el cual el Estado aporte el total o parte del valor de las primas correspondientes a la población de menores recursos, sustituyendo así el subsidio a la oferta-que actualmente brinda-por el subsidio a la demanda, lo que significa libertad de elección del usuario tanto en lo que se refiere a la entidad de aseguramiento como al prestador de los servicios. Se emplea el análisis actuarial financiero para los cálculos necesarios, el cual se sustenta en análisis de la situación de salud y de las transacciones de los servicios en el mercado en términos de sus consecuencias financieras. Uno de sus principales hallazgos es confirmar que el problema de la salud en el Perú no es la falta de recursos, sino la forma como se ha estructurado el sector salud y el sistema de subsidios.
\end{abstract}

E: cualquier país, el objetivo central del sector salud es mantener o mejorar el estado de la salud de la población. Esto se logra previniendo las enfermedades, evitando las epidemias y proveyendo servicios de curación y rehabilitación a los ciudadanos que los necesiten. Estas acciones deben llevarse a cabo tan eficientemente como sea posible y, sobre todo, a un costo que pueda ser afrontado por los actores privados y públicos, es decir, de un modo sustentable y sostenible por la sociedad.

Normalmente, el sector salud involucra a un conglomerado de instituciones públicas y privadas que cumplen diferentes funciones y que en conjunto forman el sistema nacional de salud. Éstas son: i) la que diseña la política, programas y 
normas, ii) las que proveen los servicios, iii) las que financian los servicios y iv) las que supervisan la provisión de los servicios. En el presente trabajo el énfasis se pone en el aspecto del financiamiento, con el propósito de sugerir cómo establecer un sistema de salud mediante el cual todos los ciudadanos puedan acceder a los servicios independientemente de su situación económica.

\section{El financiamiento de la salud}

El aspecto financiero de un sistema de salud puede operar bajo diversos esquemas: sistema de seguros (privados o públicos), servicios de salud públicos financiados por impuestos, pago directo del usuario por acto médico, o por una combinación de éstos. En la mayoría de ellos, dependiendo del nivel de desarrollo del país o del ingreso de las familias, el Estado destina buena parte de sus ingresos a subsidiar los servicios de salud. Ello obliga a los gobiernos, por un lado, a desarrollar mecanismo eficientes de subsidio $\mathrm{y}$, por otro lado, a los entes financiadores, como pueden ser los sistemas de seguro -públicos o privados-, a establecer sistemas de financiamiento eficientes y a enfrentar con imaginación el incremento constante de los costos de los servicios, dado el avance de la tecnología, principalmente, de diagnóstico.

En efecto, tanto los Fondos de Salud -el presupuesto del Ministerio de Saludcomo los prepagos de salud-EsSalud, las entidades prestadoras de salud, los seguros privados de salud-, que son los responsables de financiar los servicios $\mathrm{y}$, por tanto, asumen el riesgo financiero, han visto aumentados sus costos debido al avance tecnológico, que ha desarrollado complejos equipos de ayuda diagnóstica, medicamentos para cura de enfermedades catastróficas o aquellas que se originan por malos hábitos de las personas. A fin de reducir costos y ganar eficiencia, una de las propuestas que está ganando cada vez más aceptación es trasladar cierta parte del riesgo financiero a los proveedores de servicios, con lo cual se limita el abuso inducido por éstos en la utilización de los servicios y se recurre a medicinas y material médico de calidad, pero de menor costo, como son los medicamentos genéricos debidamente certificados. Todo ello se logra mediante convenios o acuerdos entre proveedores de servicios de salud y financiadores, convenios que deben ser promovidos por la institución competente del Estado, es decir, el Ministerio de Salud.

Sin embargo, en el sector salud se observan aún contradicciones notables, pues algunos ciudadanos no pueden acceder a un servicio cuando lo necesitan, mientras otras personas y ciertas instituciones hacen uso excesivo e injustificado de los servicios. Por esta razón, las autoridades de salud y los sistemas colectivos de financiamiento, sean públicos o privados, son cada vez más sensibles a estas circunstancias.

Frente a la actual escasa posibilidad de gran número de ciudadanos de recibir servicios, el Estado, además de proporcionar más fondos al sector, está en la obligación de desarrollar sistemas más eficientes de asignación de fondos. Mientras que para afrontar el constante incremento de los costos hay dos alternativas: establecer mecanismos de racionamiento de distribución de servicios, como sucede en los sistemas públicos del Perú -haciendo así aun más difícil el acceso a la salud a las 
personas más necesitadas-, o dejar que las fuerzas del mercado establezcan los precios. En este segundo caso, se crearían escenarios de mayor inequidad, pues muchos ciudadanos no podrían acceder a ciertos servicios de salud porque los precios de éstos serían mayores a los que ellos podrían pagar. Ambas alternativas generan preocupaciones éticas a los gobernantes y a la sociedad en su conjunto.

En realidad, quienes gobiernan los sistemas de salud no llegan a entender a cabalidad la interacción de tres factores importantes: la necesidad de servicios de salud de la población, la diferencia entre necesidad de servicios y demanda de servicios, y el derecho de los ciudadanos a un paquete de servicios de salud mínimo, el mismo que puede incrementarse con el tiempo y posibilidades de los gobiernos ${ }^{1}$. La relación entre el uso o consumo de servicios de salud y los niveles de salud es también poco entendido o erróneamente determinado. De todo ello se deriva gran incertidumbre entre gobernantes, políticos e instituciones de la sociedad civil.

A fin de que un país pueda generar un sistema de salud accesible y totalmente financiable para toda la población en necesidad real de servicios, los recursos deben ser usados de la mejor forma posible, bajo el concepto de intervenciones costobeneficio. Ello puede lograrse con la asignación efectiva y eficiente de los recursos actuales, dirigiéndoles adonde realmente se necesiten, y empleando mecanismos financieros de control de costos. Para que un sistema de salud sea fi-

1. En los sistemas de seguros públicos de salud los «paquetes» de servicios se denominan beneficios, mientras en los seguros privados se denominan planes o programas de salud. nancieramente sustentable, los recursos deben ser asignados de manera transparente, manejados por una gerencia financiera eficiente y empleando sistemas de monitoreo y control de las intervenciones y de los resultados de las mismas, tanto si las instituciones participantes son públicas como si son privadas o mixtas.

\section{Los modelos financieros de salud}

Uno de los elementos que ayudan a generar una correcta asignación de recursos (humanos, financieros y de infraestructura) y, por tanto, un sistema de salud sustentable financieramente son los modelos financieros de salud. Estos modelos se sustentan en dos tipos de instrumentos cuantitativos: los descriptivos y los analíticos.

Los instrumentos descriptivos son estándar y se utilizan constantemente para elaborar reportes financieros y de control en forma de registros contables y estadísticas. Sirven para mostrar resultados y la eficiencia de un gobierno o sistema que pretende ser transparente.

Los instrumentos analíticos, por su parte, permiten a los diseñadores de política «mapear» la realidad financiera y de salud de un ámbito geográfico cualquiera, método usado para hacer evaluaciones. Con estos mapas es posible determinar el monto de recursos financieros necesarios para mantener el nivel actual cuantitativo y cualitativo de los servicios de salud o hacer proyecciones para implementar mejoras en el estado de la salud.

Los resultados de estas proyecciones pueden ser usados para negociar presupuestos adicionales con la autoridad com- 
petente, elevar la presión tributaria o aumentar el nivel de las contribuciones por seguros de salud. Asimismo, pueden ser usados para establecer mejoras en los sistemas de salud, aumentar las coberturas de los planes o programas de servicios de salud o los derechos de los aportantes y ciudadanos. Igualmente pueden ser utilizados para calcular cuál sería el costo de todos los servicios de salud dirigidos a una población determinada o para proyectar el gasto futuro necesario que requeriría la atención de pacientes con enfermedades específicas -como el sida, por ejemplo-y, por lo tanto, para elaborar los presupuestos correspondientes. Es decir, se pueden generar una serie de escenarios de servicios de salud y necesidades financieras.

Los instrumentos analíticos empleados para elaborar estas proyecciones se denominan modelos y son, básicamente, formulaciones matemáticas acerca de sistemas de salud y sistemas financieros. Estos modelos necesariamente se basan en las regulaciones sobre el financiamiento de los sistemas de salud establecidos por los gobiernos y en la oferta existente de beneficios y/o planes o programas de servicios de salud. Asimismo, toman en cuenta la interacción resultante de varios factores que actúan en el mercado de la salud y variables sobre la situación económica.

A pesar de su utilidad y disponibilidad, en el Perú estos modelos son muy poco empleados o totalmente ignorados, tanto por las instituciones de planeamiento y administración del Ministerio de Salud como por las instituciones privadas de salud, foros de discusión, investigadores y tomadores de decisiones políticas. Se utilizan solamente instrumentos descrip- tivos para formular proyecciones de presupuestos, por ejemplo, o para plantear, sin mayor sustento, soluciones al poco acceso a los servicios de la población más necesitada.

\subsection{La construcción del modelo}

Un modelo presenta las características más importantes de una situación dada, ignorando aspectos poco relevantes. Se sustenta en evidencia empírica y en realidades que son la base para hacer predicciones de conductas futuras. Es construido en estrecha interacción entre las hipótesis y las observaciones.

Los modelos financieros de salud consideran entre sus variables aspectos económicos y financieros, conductas de salud de las personas y costos de los servicios. Algunos incorporan la evolución de la población y de su conducta, contribuciones y aportes y reglas financieras, llegando a ser modelos actuariales. Además, los modelos financieros de salud descansan en realidades concretas dadas, como morbilidad, mortalidad, demandas por servicios de salud y otros. Con toda esta información se elaboran proyecciones futuras sobre la necesidad de financiamiento para atender las necesidades de salud de una población determinada.

Para construir un modelo financiero de salud se deben emplear modelos previamente elaborados y realizar análisis o mapeos siguiendo el orden secuencial que se presenta a continuación:

- Modelos de la situación de salud, que utilizan todos los factores que determinan el estado de salud de una población dada, generando indicadores de salud. Éstos son normalmente cons- 
truidos por epidemiólogos, quienes buscan vincular una situación observada de salud o indicadores seleccionados a un conjunto de causas.

- Análisis o transformación de información, que relaciona algunos o todos los indicadores de salud con las transacciones en el mercado del cuidado de la salud. Este análisis vincula información epidemiológica e información económica concerniente a variables de demanda sobre el mercado de la salud. Esto es así porque la demanda por servicios de salud refleja los comportamientos de la morbilidad y los servicios agregados de prevención, curación y rehabilitación llevadas a cabo por el sistema de salud.

Es importante señalar que, por lo complicado del tema, muchas veces los planificadores de salud encuentran dificultades en cómo evaluar y responder a las variables de demanda. Las variables de demanda, obviamente, tienen un impacto sobre el total del gasto en los sistemas de salud. Sin embargo, la relación entre los bienes y servicios ofrecidos por los profesionales médicos y ciertos estados de salud o enfermedad es compleja y está basada en determinantes que consideran algo más que necesidades médicas de los pacientes.

De hecho, se acepta que las variables de demanda pueden originarse tanto por el lado de la demanda misma como por el lado de la oferta, por las particularidades de este mercado. Este tipo de análisis usualmente debe ser elaborado por profesionales de planificación de la salud, de modo que se pueda lograr una visión clara sobre el mer- cado de la salud y determinar, entre otros aspectos difíciles de discernir, cuánto de la demanda es generada por la situación de salud y cuánta es inducida por la tecnología, los profesionales de la salud y otros agentes económicos.

Al mismo tiempo, debe tenerse presente que la demanda agregada por servicios de salud es afectada por los recursos de salud disponibles y por incentivos o desincentivos desarrollados dentro del sistema de financiamiento de la salud.

- Análisis sobre las interacciones en el mercado de la salud en términos de sus consecuencias financieras, que es lo sustantivo para el desarrollo de un modelo financiero de salud. Como se mencionó anteriormente, estos modelos también toman en consideración los cambios en la disponibilidad de recursos y la posibilidad de cambios en la conducta de los proveedores de servicios de salud, los que son presionados, entre otros, por los cambios tecnológicos.

Un modelo que incluya toda la información hasta aquí descrita permitiría obtener respuestas a preguntas como las siguientes: ¿cuánto costaría financiar los servicios de salud de las personas que tienen una necesidad básica insatisfecha?, ¿cuánto costaría a los contribuyentes, empleadores y trabajadores establecer algún sistema de seguro de salud obligatorio?, ¿cuánto más dinero se requeriría si se quisiera ampliar la cobertura de seguros de salud a los trabajadores agrícolas?

En la figura 1 se aprecia una síntesis de estos análisis. 


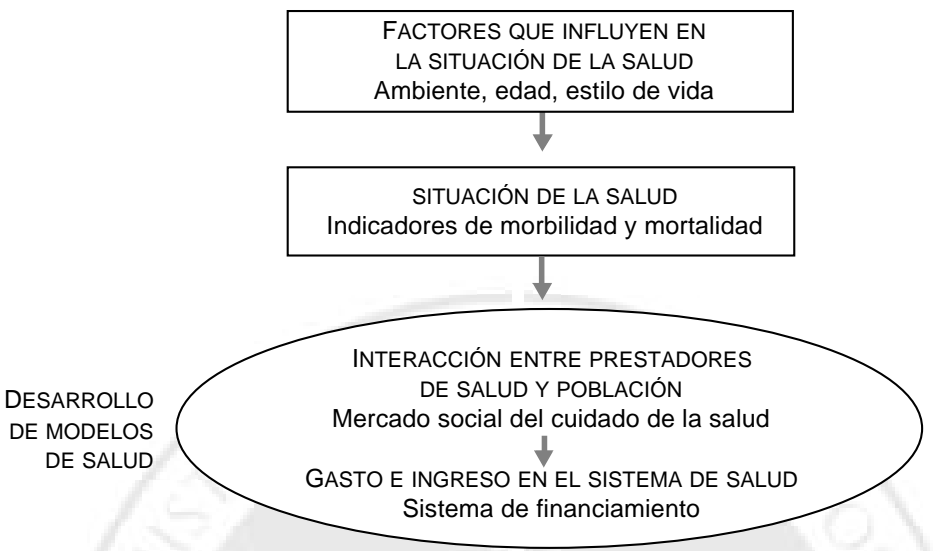

Figura 1: Los modelos y provisión de servicios

Las preguntas anteriores pueden sintetizarse en una sola: ¿cuál es el costo de los servicios de salud de un plan o programa definido para un grupo poblacional dado? Independientemente de cómo se generen los ingresos (impuestos, aportes, contribuciones, copagos) la respuesta puede hallarse con la siguiente fórmula básica:

$$
P(t)=\frac{G T B(t)+C A-I}{C T(t)}
$$

Donde:

$\mathrm{P}=$ es la prima o aporte de los asegurados y/o la tasa de contribución del Estado por persona, y que proviene de impuestos.

$\mathrm{t}=$ representa un año.

GTB $=$ es el gasto total en los beneficios o servicios de salud.

$\mathrm{CA}=$ es la suma de costos administrativos y otros costos no aplicados a beneficios.
$\mathrm{I}=\quad$ son los ingresos no provenientes de contribuciones, tales como intereses de inversiones o fondos de contingencia.

$\mathrm{CT}=$ es la contribución total, que puede provenir de impuestos, primas de seguros, copagos, deducibles, entre otros.

Esta fórmula fija cuál debe ser la contribución por persona en un sistema de salud dado, independientemente que el aporte sea hecho por el individuo o por el Estado a manera de subsidio; igualmente, muestra el equilibrio financiero del sistema, es decir, ingresos y gastos son iguales.

Debe tenerse presente que detrás de esta ecuación están los factores de producción de los servicios de salud, esto es, recursos humanos, medicinas, infraestructura de salud, entre otros, que son financiados en su totalidad por la contribución total. 


\subsection{El contexto económico y social en la construcción del modelo}

Ahora bien, antes de desarrollar un modelo en su total capacidad, es importante entender cómo funciona un sistema de salud en una situación dada o en un país en particular. En otras palabras, es importante comprender los mecanismos por los cuales la necesidad de un servicio de salud se convierte en demanda por el servicio y, por tanto, se concreta en un gasto de salud en un esquema determinado.

Se puede asumir que hay una relación entre la situación de la salud y el consumo de servicios de salud, aun si el beneficio marginal declina a altos niveles de consumo. También es obvio que hay una relación entre el nivel de ingreso y la cantidad e intensidad de los servicios de salud consumidos. La situación de salud determina la necesidad por servicios de salud, pero no necesariamente en todos los casos determina la demanda por servicios de salud, ya que hay gente de bajos ingresos que no pueden pagar por estos servicios y, por lo tanto, no los demandan.

Estos mecanismos tienen dos implicancias primordiales para el diseño de modelos de salud. En primer lugar, se puede asumir que las tasas de utilización están relacionadas con los niveles de ingreso, lo que puede ser expresado por la elasticidad ingreso de demanda por servicios de salud. En segundo lugar, esta elasticidad no es constante a todos los niveles de ingreso; es más alta mientras más altos son los ingresos. En cambio, la demanda potencial es alta en las personas de bajos ingresos porque su situación de salud es realmente precaria; por ello, cuantos más recursos estén disponibles en los sistemas de salud, mayor será el incremento en la utilización de los servicios de salud por parte de la gente pobre en comparación con las personas de ingresos altos.

Otros factores que son identificables en el consumo de servicios de salud son la edad y el sexo. Poblaciones con diferentes edades tienen diferentes situaciones de salud y, por tanto, necesidades de atención diferentes. En lo que se refiere al sexo, si bien la situación de salud puede ser similar a la misma edad, no sucede lo mismo con las necesidades de atención, dadas las condiciones naturales de la mujer y el hombre. La determinación precisa de programas de beneficios o programas de salud por grupos de personas hace más viable las posibilidades de financiamiento de los servicios de salud para una mayor cantidad de personas $y$, por qué no, para toda la población con reales necesidades de servicios.

Los tipos y cantidades de servicios consumidos por sexo, edad y por realidades geográficas diferentes pueden ser determinados estadísticamente. Así, se puede establecer cuántas consultas por persona al año se realizan, cuántas por sexo y edad, cuál es el ratio de hospitalizaciones por consulta, cuántos análisis de laboratorios se hacen por cada cien consultas, etc. Todos estos datos establecen que si hay cambios en la situación de salud o en el esquema de beneficios, la demanda agregada de servicios cambiará. También se puede establecer qué cantidad adicional de recursos de salud se requiere para atender el incremento de la demanda efectiva si cambia el sistema de beneficios o se incorpora a éste mayor población.

El ingreso, por otro lado, es determinado en gran medida por el presupuesto asignado al sector por el Estado -prove- 
niente de impuestos- o por las contribuciones de los aportantes a un sistema de seguros. Ello significa que el equilibrio financiero de los diferentes esquemas de salud puede ser afectado por la condición económica de un país. Por ejemplo, si el PBI o el nivel de empleo se reducen, el ingreso fiscal puede disminuir, así como el número y cantidad de aportantes a esquemas de seguros, lo que significará menores ingresos para el sector, pero no necesariamente que la cantidad de beneficios y beneficiarios deba reducirse.

Lo anterior no es una lista exhaustiva de todos los elementos que influyen en el equilibrio financiero del sector salud, pero sí demuestra que el financiamiento del cuidado de la salud opera en un contexto económico y social cuyas variables pueden y deben ser identificadas con deteni- miento y trasladadas a un esquema o modelo concreto de financiamiento de la salud. Estos elementos pueden, grosso modo, ser agrupados en cuatro categorías:

- Factores demográficos y laborales, tales como crecimiento y cambios en la estructura poblacional y en la población económicamente activa.

- Factores económicos, tales como desempleo, niveles de ingresos y salarios, precios y tasas de interés.

- Situación de la salud, tecnología médica y prácticas médicas, tales como tasa de morbilidad, nuevos equipos de diagnóstico, entre otros.

- Factores de gobierno, tales como decisiones respecto a quiénes tienen derecho a cobertura de servicios gratuita, quiénes están obligados a copagos, qué servicios son cubiertos, entre otros.

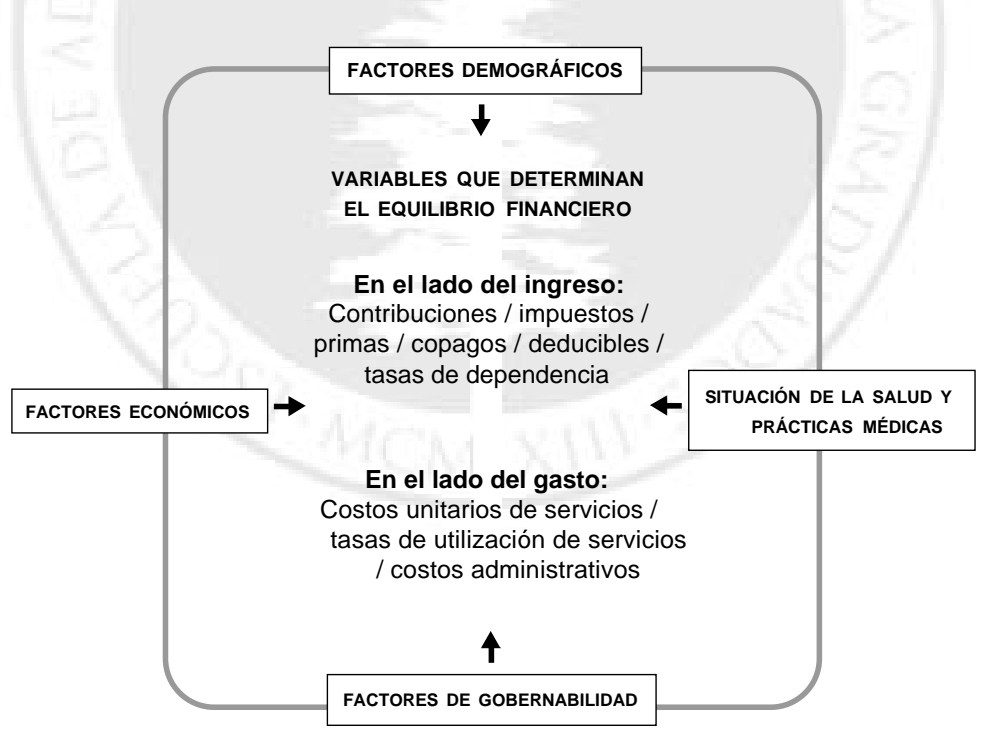

Figura 2: Equilibrio financiero de un esquema de salud 
La figura 2 muestra un esquema de financiamiento de la salud en el que se aprecia su interacción con el medio económico, social, demográfico y de decisiones gubernamentales en el que está inscrito.

\section{Un modelo de financiamiento de salud para el Perú}

Entre los factores de gobierno que forman parte del contexto económico y social en el que se inscribe el financiamiento de la salud están básicamente los objetivos de salud que se plantean lograr en periodos de tiempo determinados. Por ejemplo, en un primer periodo, puede ser reducir la morbilidad y mortalidad infantil, luego dar cobertura gratuita de servicios de salud a escolares y madres gestantes, después continuar con un programa de cobertura total para los enfermos de SIDA y tuberculosis, y así sucesivamente.

También cae dentro de las decisiones gubernamentales el cómo se elija cumplir estos objetivos; es decir, a través de qué tipo de instrumentos u organización del sistema de salud. En el Perú, que se desenvuelve en un esquema de economía social de mercado, se ha establecido que con fondos del Estado se debe financiar los servicios de salud de las personas que por sus niveles de ingreso no pueden hacerlo. El financiamiento de los gastos de salud de las personas de mayores recursos corre por cuenta de ellas mismas, ya sea por pago por acto médico o por pago de primas o aportes a entidades de prepago.

En el Perú, para el caso de las personas que disponen de recursos para afrontar sus gastos de salud, se han ido desarrollando una serie de esquemas de seguros con autorización o sin ella del Minis- terio de Salud o de la autoridad competente. Los principales son los siguientes:

a) El Seguro Social de Salud, EsSalud.

b) Las entidades prestadora de salud, EPS.

c) Las compañías de seguros que ofrecen programas o planes de salud.

d) Las empresas o instituciones que desarrollan para sus trabajadores planes o programas de salud.

e) Las clínicas o establecimientos de salud que ofrecen programas o planes de salud al público en general.

f) Los organismos no gubernamentales que ofrecen programas o planes de salud al público de menores recursos.

g) Los organismos de gobierno que ofrecen programas o planes de salud al público en general.

De todos estos esquemas, solamente las entidades prestadoras de salud cuentan con una legislación completa, que a la vez regula su funcionamiento y protege a sus afiliados.

En el caso de las personas de bajos recursos, el gobierno está paulatinamente sustituyendo el esquema de subsidio a la oferta por el esquema de subsidio a la demanda, a través de lo que se ha denominado Seguro Integral de Salud, SIS. Esto significa que con el correr de los años el pliego presupuestal del Ministerio de Salud ya no estará formado por la planilla de médicos, las inversiones en establecimientos de salud, la compra de medicamentos, etc., sino que será resultado de multiplicar el valor del aporte o prima promedio por año por persona por la población beneficiaria. Es decir, responderá a la siguiente fórmula:

$$
P(t) * N=G T B(t)+C A-I
$$


donde $\mathrm{N}$ indica el número de beneficiarios.

Sin embargo, con el transcurso del tiempo también se tendrá que establecer que el nivel de subsidio de salud no deberá ser igual para todos los beneficiarios, sino que deberá estar en función del nivel de ingreso de cada uno de ellos. Por ejemplo, se podría señalar que para una persona con tres o más necesidades básicas insatisfechas, NBI, el subsidio deberá ser del $100 \%$, para una persona que tiene dos necesidades insatisfechas el subsidio deberá ser $50 \%$ y así sucesivamente. Estos aspectos son decisiones de política que tendrían que tomarse teniendo en cuenta los recursos disponibles y el objetivo de eficiencia perseguido.

Considerado todo lo señalado hasta ahora, a continuación se desarrolla un modelo que permitirá establecer las necesidades de financiamiento de la salud para personas de escasos recursos, que por ahora son subsidiados de manera mixta, a través del Seguro Integral de Salud y la oferta de servicios de los establecimientos de salud públicos. El modelo que se propone corresponde a un sistema de seguros de salud, pero no es semejante al actual Seguro Integral de Salud -que sin duda a la larga presentará los mismos vicios de EsSalud-, se basa, más bien, en entidades de prepago que surjan bajo el amparo de la ley y a las cuales las personas se afiliarán de acuerdo con sus preferencias. Sólo mediante una modalidad de esta naturaleza se podrá lograr un sistema financiero adecuadamente estructurado (costobeneficio) que asigne los recursos humanos, financieros, equipos, infraestructura, entre otros, de una manera racional y eficiente. Es decir, que dirija los recursos donde hay necesidad de servicios y no como ahora, que se dirigen donde hay infraestructura hospitalaria, con frecuencia arbitrariamente construida.

A fin de garantizar el derecho social fundamental a la salud, hace falta un seguro que otorgue sus prestaciones no en función de las cotizaciones (que dependen de los ingresos de las personas), sino en función de las necesidades (derivadas del estado de la salud); es decir, un sistema que subsidie a las personas de menores ingresos y que cubra total o parcialmente, según el caso, el valor del aporte al seguro. Para ello, el Estado recurre a los fondos recaudados por impuestos.

Mediante el mecanismo del seguro, todas las personas tendrían que afiliarse a un seguro de salud. Las personas pudientes pagarían ellos mismos el costo de la prima, mientras los pobres serían total o parcialmente subsidiados por el Estado ${ }^{2}$. El financiamiento de la salud se haría a través de entidades de prepago, las cuales pagarían a los establecimientos de salud por el tratamiento de sus afiiados, ya sea por capitación, acto médico o cualquier otra modalidad. De esta manera se estaría garantizando uno de los principios fundamentales de la salud: la libre elección, ya que cada persona decidiría a qué entidad afiliarse, independientemente de su situación económica y capacidad de pago, y en qué lugar atenderse.

2. El financiamiento parcial de los seguros de salud de las personas de más bajos ingresos no es nuevo en el Perú. Debe recordarse que cuando se crearon el Seguro del Obrero y del Seguro del Empleado, que luego se fusionaron, una parte de los aportes la proporcionaba el Estado, pues se entendió que el descuento del salario para el aporte mensual al seguro no cubría, en muchos casos, el costo de la prima. 
Un sistema de financiamiento de la salud concebido de esta manera asignaría mejor los escasos recursos, pues cuando el Estado establezca que todos los ciudadanos deberán inscribirse en una entidad de prepago y que la prima de los menos pudientes la pagará el Estado, el dinero fluirá hacia donde hagan falta servicios de salud, no como hasta ahora, que se concentra adonde hay más médicos o más infraestructura.

Por ejemplo, sí los ciudadanos de un área marginal de la sierra se inscriben en una entidad de prepago creada en ese lugar, el Estado tendrá que dirigir dinero hacia esa localidad en un monto tal que cubra los programas de salud diseñados para esas personas. Ese dinero será administrado por esa entidad de prepago y funcionará como un incentivo para que se creen establecimientos de salud apropiados en la localidad, pues los prestadores estarán seguros de que cuando atiendan a un usuario afiliado al sistema, la entidad de prepago pagará por los servicios.

Un esquema de esta naturaleza generaría la economía social de mercado en el sector salud.

Antes de pasar a desarrollar el modelo planteado es importante conocer cuáles son los principios básicos de un seguro, que es el mecanismo financiero adoptado para el financiamiento de la salud por ser el de mayor costo-beneficio, como se ha explicado líneas arriba.

Los ingresos de los seguros son las contribuciones o aportes de los afiliados, sean hechas por ellos mismos o mediante subsidios del Estado. El pago o aporte (prima de seguro) que se hace mensualmente es en realidad un prepago de dine- ro que le permite al aportante o afiliado protegerse ante una catástrofe financiera que tenga que afrontar en el futuro por algún siniestro que le pueda ocurrir.

En el caso de los seguros de salud, ya sea que el aporte sea hecho a EsSalud, a una EPS, a un seguro privado o al Seguro Integral de Salud, la situación y las características son las mismas. Los afiliados o aportantes prepagan dinero para protegerse de la catástrofe financiera que tendrían que afrontar si les sucede un accidente o contraen una enfermedad.

El aporte o prepago que en conjunto hacen los afiliados o aportantes debe ser de una magnitud tal que permita a la empresa de seguro o al sistema financiar las enfermedades o accidentes de todos los afiliados que sufran siniestros durante un mes o un año, según se hagan los cálculos. En consecuencia, cada empresa de seguros que ofrece pólizas de salud tiene que determinar el valor del aporte o prima de cada persona o familia en función del número de afiliados que pueda conseguir y el programa de servicios de salud a que los afiliados tengan derecho.

El punto crucial de todo seguro, en este caso de un seguro de salud, reside básicamente en determinar el aporte o prima mensual que el afiliado debe pagar. Para ello debe conocerse:

- Cuál es el número probable de siniestros que habrá en un mes determinado dentro de una población determinada. Es decir, de 100 personas afiliadas cuántas se enfermarán, cuántas solicitarán consultas, cuántas requerirán medicinas, cuántas serán hospitalizadas, etc. Estos datos los establecen los planificadores y estadísticos de salud. 
- Cuánto cuestan en promedio los tratamientos, que incluyen consulta, medicinas, laboratorio, rayos $\mathrm{X}$, hospitalización, etc., por tipo de enfermedad.

La suma de los gastos necesarios para atender a los enfermos se denomina siniestralidad. A este monto se le añade los gastos de administración del sistema, la reserva de contingencia y la probable utilidad.

El análisis actuarial financiero es la herramienta que se utiliza para i) determinar cuál es el valor de la prima o aporte por afiliado o beneficiario, ii) conocer las necesidades de financiamiento de todo el sistema para atender a toda la población con derecho a los servicios y iii) el copago, que es el valor que desembolsa el afiliado cuando busca una atención. Este copago puede ser el instrumento mediante el cual el Estado diferencie el nivel de subsidio por persona.

Para el cálculo de la prima o aporte se ha desarrollado el siguiente modelo ${ }^{3}$ :

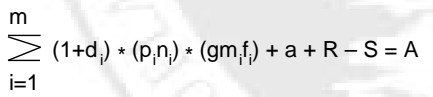

Donde:

$\mathrm{m}=$ representa las enfermedades o males de salud que pueden afectar al ser humano a lo largo de su vida. Estos datos los elaboran los epidemiólogos.

$1+d=e s$ el coeficiente de seguridad, que se calcula a partir de la variación promedio del costo de cada trata-

3. En los seguros privados de salud se denomina prima de riesgo a $(1+\mathrm{di}) *$ pini $*(\mathrm{gmi})$, mientras que la prima neta vendría a ser AT. miento. Es decir, la desviación estándar de los gastos de todos los pacientes en un periodo de tiempo.

$\mathrm{p}=$ representa la probabilidad de ocurrencia de la enfermedad por sexo y edad. Sin embargo, en salud se recurre al acto médico por enfermedad, dado que estos actos varían sustancialmente de persona a persona. Estos datos deben elaborarlos los planificadores de salud o estadísticos.

$\mathrm{n}=$ representa el número de afiliados que tendrá el seguro, es decir, las personas con derecho a servicios del plan que se establezca, independientemente que sean subsidiados o no.

$\mathrm{g}=$ representa el gasto por cada enfermedad o tratamiento, datos que se toman del tarifario de un sistema de salud. En este caso se están utilizando los tarifarios de los establecimientos de salud públicos.

$f=$ representa la frecuencia de uso de cada servicio; por ejemplo, número de análisis por consulta.

$\mathrm{a}=$ es el costo de la administración del programa, que incluye auditorías médicas y costos operativos.

$\mathrm{R}=$ es el margen de seguridad, que se sustenta en el valor presente de los ingresos y gastos asumiendo una tasa de interés actuarial, dado que los cálculos se hacen por periodos largos de tiempo. Ello, porque los seguros se calculan a partir de los fondos que aportan los afiliados cuando son jóvenes. Por tanto, la prima es más alta que el costo de los beneficios en que ellos incurren, lo que no sucede cuando estas mismas personas envejecen. 
$\mathrm{S}=$ es el cálculo de las reservas por las personas que se retiran del sistema de seguros, ya sea por alguna circunstancia (viaje, cambio de seguro, etc.) o por muerte. Esto se establece actuarialmente a partir de la probabilidad de muerte y la probabilidad de cancelación.

$\mathrm{A}=$ es la prima de riesgo, es decir, lo que debe aportar cada individuo para tener cobertura en el programa de salud.

En los seguros de salud privados se trata de controlar el excesivo uso de los servicios por medio del copago, mientras en los seguros públicos, además de perseguir el mismo propósito con mecanismos similares, se busca diferenciar los niveles de subsidio otorgados a cada persona de acuerdo con su nivel socioeconómico. Por ello, en los seguros de salud y según la estructura de financiamiento del plan o programa específico, otras variables tienen que formar parte del análisis actuarial financiero. Éstas son:

$\mathrm{c}=$ el copago o deducible por servicios, que es el precio que pagan algunos asegurados al recibir determinados servicios.

$\mathrm{C}=$ La cobertura, que determina hasta qué porcentaje del costo de la atención cubre el seguro y qué parte tiene que pagar el asegurado.

De este modo, la fórmula inicial queda de la siguiente manera:

$\sum_{i=1}^{m}\left(C_{i} p_{i}\right) *\left\{\left\{\left(g m_{i}\right) *\left(1+d_{i}\right)\right\} * c\right\}(n)+a+R-S=A$

Luego, con el propósito de conocer cuál es el monto total de recursos finan- cieros (IT) que requiere el sistema para atender a una población determinada, la prima de riesgo (A) tiene que ser multiplicada por el número de individuos cubiertos (n).

\section{Entonces:}

$$
\mathrm{IT}=\mathrm{A} * \mathrm{n}
$$

El modelo se ha desarrollado en hoja de cálculo e incluye solamente la primera parte de la fórmula:

$$
\left(C_{i} p_{i}\right) *\left\{\left\{\left(g m_{i} f_{i}\right) *\left(1+d_{i}\right)\right\} * c\right\}(n)
$$

Los resultados se muestran en el cuadro 1, que resume el análisis actuarial para personas con tres o más necesidades básicas insatisfechas (NBI). Este ejercicio se ha hecho utilizando información gruesa, pero es una aproximación muy cercana al monto que debería presupuestar el Minsa para financiar los servicios de salud curativos del grupo poblacional que debería tener derecho al subsidio por el $100 \%$ del costo de los servicios. Como ya se ha señalado, el mecanismo del subsidio será el copago; por lo tanto, en el primer ejercicio del modelo este rubro será cero.

En el cuadro, la primera columna contiene los diferentes tipos de servicios, es decir, el plan de servicios de salud o programa de beneficios al cual el grupo poblacional señalado tendría derecho, mientras en las siguientes columnas se han colocado todas las variables que exige la fórmula utilizada. Se presenta información desagregada por consulta, internamiento, acto quirúrgico, cirugía de día y emergencia, entre los más representativos, así como medicinas, laboratorio y rayos $\mathrm{X}$.

El ejercicio no contiene una desagregación por grupos de edad o género, pues la información disponible y a la que se 
puede acceder no permite hacer un análisis más detallado; sin embargo, los parámetros de consumo de servicios que se han usado representan el promedio de consumo de toda la población.

La sumatoria al final del cuadro determina la siniestralidad anual de una persona afiliada. A este valor se le debe añadir los otros elementos de la fórmula, los cuales son calculados exógenamente: $\mathrm{a}+\mathrm{R}-\mathrm{S}$. El gasto operativo, que representa el gasto en seguimiento o auditoría médica, más el gasto administrativo, son datos que se pueden obtener de la administración del Minsa, que en este ejercicio se asume es de $10 \%{ }^{4}$. La reserva (R) y las salidas de afiliados (S) no se han considerado porque los cálculos están referidos a un año y corresponden al monto que debería asignar el MEF al Minsa para este propósito.

Para el cálculo de las necesidades de financiamiento correspondientes a la población con tres y más necesidades básicas insatisfechas se ha utilizado la siguiente información:

- Los planes y cobertura que establece el SIS, incluyendo para este ejercicio a población de toda edad.

- Las probabilidades de uso del servicio que ya estableció el SIS en sus primeros años de funcionamiento. Por coincidencia, estas probabilidades son similares a los ratios que se obtienen de EsSalud.

4. En diversas empresas de prepago se asume que un gasto administrativo de $10 \%$ es lo razonable. Cualquier porcentaje mayor a éste es considerado como un excesivo gasto burocrático. Algunas instituciones privadas, EsSalud entre ellas, establecen ese $10 \%$ de gasto administrativo en sus estatutos.
- La frecuencia de uso de servicios del SIS.

- El costo de los servicios o tarifario de los establecimientos públicos. El dato que se incluye en el modelo considera tanto la parte del costo que corresponde al subsidio a la demanda como aquella que corresponde al subsidio a la oferta, a través de los salarios de los profesionales de la salud.

- Para el cálculo de la desviación estándar se ha tomado la variación de precios de los tratamientos en el sector privado.

El resultado, una siniestralidad anual de 875,91 soles es el costo técnico de la prima anual por persona, lo que equivale a 72,99 soles mensuales, sólo por servicios de curación y rehabilitación. No incluye prevención ni el costo de otros bienes o servicios públicos de salud.

Si a este monto se le agrega el $10 \%$ por concepto de gastos administrativos, el costo de la prima por persona al año asciende a 948,90 soles.

De acuerdo con datos de la ENAHO 2001 del IV trimestre, la población con tres o más necesidades básicas insatisfechas es de aproximadamente 2,5 millones, cifra que multiplicada por 948,90 soles indica que se necesita casi dos mil trescientos millones de soles para financiar la curación y rehabilitación de ese grupo de personas que no pueden pagar por servicios curativos de salud.

Como se ha señalado, la primera columna del cuadro representa el programa o paquete de servicios del SIS. A este paquete se puede añadir los servicios pre- 
Cuadro 1

Análisis actuarial del SIS: servicios de capa simple Población con una necesidad básica insatisfecha (NBI)

\begin{tabular}{|c|c|c|c|c|c|c|c|}
\hline Servicios & Deducible & Cobertura & Frecuencia & Probabilidad & $\begin{array}{l}\text { Precio en } \\
\text { soles }\end{array}$ & $\begin{array}{l}\text { Desviación } \\
\text { estándar }\end{array}$ & Siniestralidad \\
\hline \multicolumn{8}{|l|}{ Egresos } \\
\hline Consultas por caso & & & 1,6274 & & & & \\
\hline 1a. consulta por caso & 0,00 & $100 \%$ & 1 & 1,500000 & 15,00 & 1 & 22,50 \\
\hline 2a. consulta por caso & 0,00 & $100 \%$ & 0,4019 & 1,500000 & 15,00 & 1,07735772 & 9,74 \\
\hline 3a. consuta por caso & 0,00 & $100 \%$ & 0,2255 & 1,500000 & 15,00 & 1,05157181 & 5,34 \\
\hline Consulta/farmacia/caso & 0,00 & $100 \%$ & 1 & 2,430000 & 90,32 & 1,13234636 & 248,52 \\
\hline Consulta /laboratorio/caso & 0,00 & $100 \%$ & 1 & 2,430000 & 14,96 & 1,19393067 & 43,40 \\
\hline Consulta/rayos X/caso & 0,00 & $100 \%$ & 1 & 2,430000 & 6,96 & 1,23053978 & 20,81 \\
\hline Consulta/otros servicios/caso & 0,00 & $100 \%$ & 1 & 2,430000 & 14,59 & 1,19883318 & 42,50 \\
\hline Promedio estancia & 0,00 & $100 \%$ & 3,96 & 0,093500 & 190,00 & 1,15226887 & 81,06 \\
\hline Hospitalización promedio/médico & 0,00 & $100 \%$ & 1 & 0,093500 & 224,38 & 1,09726175 & 23,02 \\
\hline Hospitalización promedio/medicinas & 0,00 & $100 \%$ & 1 & 0,093500 & 712,76 & 1,10847409 & 73,87 \\
\hline Hospitaliazación promedio/laboratorio & 0,00 & $100 \%$ & 21,10914736 & 0,093500 & 7,58 & 1,16881907 & 17,48 \\
\hline Hospitalización promedio/rayos X & 0,00 & $100 \%$ & 1,774540244 & 0,093500 & 34,44 & 1,30800502 & 7,47 \\
\hline Hospitalización promedio/otros servicios & 0,00 & $100 \%$ & 1,061858132 & 0,093500 & 241,03 & 1,42266716 & 34,04 \\
\hline Hospitalización promedio/intervención quirúrgica & 0,00 & $100 \%$ & 0,401958443 & 0,093500 & 2001,85 & 1,13307014 & 85,25 \\
\hline Hospitalización promedio/sala recuperación & 0,00 & $100 \%$ & 0,401958443 & 0,093500 & 95,96 & 1,10605389 & 3,99 \\
\hline Hospitalización promedio/otros & 0,00 & $100 \%$ & 1 & 0,093500 & 223,11 & 1,127654 & 23,52 \\
\hline Emergencia/médico/caso & 0,00 & $100 \%$ & 1 & 0,560000 & 30,21 & 1,09242435 & 18,48 \\
\hline Emergencia/farmacia/caso & 0,00 & $100 \%$ & 1 & 0,560000 & 100,02 & 1,1481366 & 64,31 \\
\hline Emergencia/laboratorio/caso & 0,00 & $100 \%$ & 1 & 0,560000 & 9,40 & 1,09558094 & 5,77 \\
\hline Emergencia/rayos X/caso & 0,00 & $100 \%$ & 1 & 0,560000 & 13,51 & 1,09557043 & 8,29 \\
\hline Emergencia/otros servicios/caso & 0,00 & $100 \%$ & 1 & 0,560000 & 4,68 & 1,14026986 & 2,99 \\
\hline Transporte evacuación & 0,00 & $100 \%$ & 1 & 0,000060 & 340,00 & 1,0002 & 0,02 \\
\hline Tratamiento odontológico & 0,00 & $90 \%$ & 1 & 0,031000 & 137,41 & 1,09169034 & 4,19 \\
\hline Tratamiento oftalmológico & 0,00 & $90 \%$ & 1 & 0,016276 & 46,85 & 1,09169034 & 0,75 \\
\hline Maternidad & 0,00 & $100 \%$ & 1 & 0,021764 & 1130,87 & 1,10331608 & 27,16 \\
\hline Servicio domiciliario/médico & 0,00 & $90 \%$ & 1,55 & 0,000310 & 33,73 & 1,12892954 & 0,02 \\
\hline Servicio domiciliario/laboratorio/otros & 0,00 & $100 \%$ & 1,55 & 0,000310 & 15,19 & 1,19393067 & 0,01 \\
\hline Servicio domiciliario/farmacia-clínica & 0,00 & $100 \%$ & 1,55 & 0,000310 & 155,51 & 1,13234636 & 0,08 \\
\hline Cirugía ambulatoria/médico & 0,00 & $90 \%$ & 1 & 0,001800 & 568,94 & 1,09726175 & 1,01 \\
\hline Cirugía ambulatoria/farmacia-clínica & 0,00 & $90 \%$ & 1 & 0,001800 & 155,51 & 1,10847409 & 0,28 \\
\hline Cirugía ambulatoria/laboratorio/otros servicios & 0,00 & $90 \%$ & 1 & 0,001800 & 15,19 & 1,16881907 & 0,03 \\
\hline Siniestralidad & & & & & & & 875,91 \\
\hline
\end{tabular}


ventivos y calcular lo que esto costaría; de esta manera se sabría cuánto dinero requiere el Minsa para atender la prevención, curación y rehabilitación de los pobres del Perú. En el presupuesto del Minsa y en los presupuestos de los gobiernos regionales -en la parte que corresponde a salud- a la prevención se le denomina salud colectiva. En este rubro, lo presupuestado durante los tres últimos años es de aproximadamente 384 millones, lo que en promedio equivale a alrededor del $18 \%$ del total del presupuesto para el sector de salud pública.

En consecuencia, si a la cifra de casi dos mil trescientos mil millones de soles obtenida anteriormente se le suma el $18 \%$ que corresponde a prevención, el monto se eleva a casi dos mil setecientos millones de soles.
El presupuesto del sector de salud pública (Minsa y gobiernos regionales) para el 2003 fue algo más de dos mil millones de soles, monto que equivale realmente a casi el $80 \%$ de lo que realmente se requiere para subsidiar a ese grupo poblacional. Este resultado es muy importante, pues confirma que el problema de la salud en el país no es la falta de recursos, sino la forma como se ha estructurado el sector salud y el sistema de subsidios.

Como se indicó anteriormente, es decisión del Estado establecer a qué grupo poblacional desea subsidiar y con qué paquete de servicios, lo que fundamentalmente se determina por la disponibilidad de fondos.

Teniendo esto en mente, a continuación se presenta un cuadro que resume

\section{Cuadro 2 \\ Seguro público de salud para el Perú Alternativas de programas de beneficios y población objetivo}

\begin{tabular}{|l|l|c|c|}
\hline \multicolumn{1}{|c|}{$\begin{array}{c}\text { Programa } \\
\text { de servicios }\end{array}$} & $\begin{array}{c}\text { Grupo poblacional } \\
\text { al cual se subsidia }\end{array}$ & $\begin{array}{c}\text { Porcentaje del } \\
\text { subsidio }\end{array}$ & $\begin{array}{c}\text { Recursos } \\
\text { necesarios en soles }\end{array}$ \\
\hline $\begin{array}{l}\text { Servicios de prevención, } \\
\text { curación y rehabilitación. }\end{array}$ & $\begin{array}{l}\text { Población con tres o más } \\
\text { necesidades insatisfechas. }\end{array}$ & $100 \%$ & 2692484140,98 \\
\hline $\begin{array}{l}\text { Servicios de prevención, } \\
\text { curación y rehabilitación. }\end{array}$ & $\begin{array}{l}\text { Población con tres y dos } \\
\text { necesidades insatisfechas. }\end{array}$ & $\begin{array}{l}100 \% \text { para 3 NBI y } \\
50 \% \text { para 2 NBI }\end{array}$ & 4471773815,98 \\
\hline $\begin{array}{l}\text { Servicios de prevención, } \\
\text { curación y rehabilitación. }\end{array}$ & $\begin{array}{l}\text { Población con tres, dos y y } \\
\text { una necesidad insatisfecha. }\end{array}$ & $\begin{array}{l}100 \% \text { para 3 NBI, } \\
50 \% \text { para 2 NBI y } \\
20 \% \text { para 1 NBI. }\end{array}$ & 5649008115,98 \\
\hline $\begin{array}{l}\text { Servicios de prevención, } \\
\text { curación y rehabilitación, } \\
\text { excluidos servicios } \\
\text { dentales, oftalmológicos, } \\
\text { traslados, emergencias, } \\
\text { servicios a domicilio. }\end{array}$ & $\begin{array}{l}\text { Población con tres o más } \\
\text { necesidades insatisfechas. }\end{array}$ & $100 \%$ & 2235421336,88 \\
\hline
\end{tabular}


varias alternativas de programas de beneficios y población objetivo. Como se puede observar, se está proponiendo una política de subsidios diferenciados según el nivel socioeconómico de la población. En la alternativa de mayor co- bertura se estaría subsidiando a toda la población que presenta por lo menos una necesidad básica insatisfecha, lo que significaría algo más del doble de lo que actualmente gasta el sector público en salud.

\section{Referencias bibliográficas}

FRANCKE, Pedro. 2001. Focalización del gasto público en salud en el Perú. Proyecto Análisis Independiente del Presupuesto Público.

- 2003. Evaluación de la ejecución del presupuesto del Ministerio de salud del año 2002. Proyecto Análisis Independiente del Presupuesto Público 2003.

JARAMILLO, Miguel y otros. 2002. Evaluación del Seguro Escolar Gratuito y Seguro Materno Infantil de la Unidad del Seguro Integral de Salud. Instituto APOYO.

\section{PERÚ. INSTITUTO NACIONAL DE ESTA-} DÍSTICA E INFORMÁTICA. Encuesta Nacional de Hogares ENAHO). Lima, INEI. Años 1997 al 2001.

—. Programa MECOVI. 2002 Condiciones de vida en el Perú: evolución 1997-2001. Lima, INEI.

MINISTERIO DE ECONOMÍA Y FINANZAS. 2003.Transparencia de cifras presupuestales. www.mef.gob.pe (dic).
PERÚ. MINISTERIO DE SALUD. 2002. Sistema Nacional Coordinado y Descentralizado de salud (SNCDS) y Seguro Integral de Salud. Boletín informativo. SIS. Lima.

PFALLER, Alfred. [1997]. El concepto de la economía social del mercado y la nueva de-civilización del capitalismo en Europa. Bonn: Fundación Friedrich. http:// library.fes.de/pdf-files/stabsabteilung/ 00071.pdf.

VERA LA TORRE, José Carlos. 2004. Una propuesta de reforma del sector salud. Perú Económico (Apoyo). Lima, marzo, págs.3-4.

\section{Economía Social de mercado en} los sectores sociales: una propuesta de reforma de Estado. Lima: ESAN. 252 págs.

2003. Cobertura y financiamiento del Seguro Integral de Salud en el Perú. Lima: ForoSalud. 39 págs. 